\title{
A CREB1-miR-181a-5p loop regulates the pathophysiologic features of bone marrow stromal cells in fibrous dysplasia of bone
}

\author{
Yu Fu ${ }^{1,2,3^{*}}$, Zhili Xin ${ }^{1,3}$, Ziji Ling $^{1,3}$, Hanyu Xie ${ }^{1,3}$, Tao Xiao ${ }^{1,3,4}$, Xin Shen ${ }^{1,3}$, Jialin Lin ${ }^{1}$, Ling Xu ${ }^{1}$ and \\ Hongbing Jiang ${ }^{1,2,3^{*}}$ (D)
}

\begin{abstract}
Background: Fibrous dysplasia (FD) is a bone marrow stromal cell (BMSC) disease caused by activating mutations of guanine nucleotide-binding protein alpha-stimulating activity polypeptide (GNAS) and is characterized by increased proliferative activity and disrupted osteogenesis of BMSCs. However, the molecular mechanisms regulating the pathophysiologic features of BMSCs in FD remain unknown. This study aimed to identify and verify the roles of the CREB1miR-181a-5p regulatory loop in FD pathophysiology.
\end{abstract}

Methods: MicroRNA (miRNA) sequencing analysis was used to identify the possible miRNAs implicated in FD. The proliferation, apoptosis, and osteogenic differentiation of BMSCs, as well as the osteoclast-induced phenotype, were measured and compared after exogenous miR-181a-5p transfection into FD BMSCs or miR-181a-5p inhibitor transfection into normal BMSCs. Chromatin immunoprecipitation and luciferase reporter assays were performed to verify the interactions between CREB1 and miR-181a-5p and their effects on the FD pathological phenotype.

Results: Compared to normal BMSCs, FD BMSCs showed decreased miR-181a-5p levels and exhibited increased proliferative activity, decreased apoptotic capacity, and impaired osteogenesis. FD BMSCs also showed a stronger osteoclast activation effect. miR-181a-5p overexpression reversed the pathophysiologic features of FD BMSCs, whereas miR-181a-5p suppression induced an FD-like phenotype in normal BMSCs. Mechanistically, miR-181a-5p was the downstream target of CREB1, and CREB1 was posttranscriptionally regulated by miR-181a-5p.

Conclusions: Our study identifies that the interaction loop between CREB1 and miR-181a-5p plays a crucial role in regulating the pathophysiologic features of FD BMSCs. MiR-181a-5p may be a potential therapeutic target for the treatment of FD.

Keywords: CREB, miR-181a-5p, Bone marrow stromal cells, Fibrous dysplasia, Proliferation, Apoptosis

\section{Background}

Fibrous dysplasia (FD) is a nonhereditary bone disease caused by GNAS gene mutation in bone marrow stromal cells (BMSCs); in FD, normal bone tissue is replaced by

\footnotetext{
*Correspondence: siyu_528@163.com; jhb@njmu.edu.cn

1 Jiangsu Province Key Laboratory of Oral Diseases, Nanjing Medical

University, No.136, Hanzhong Road, Nanjing 210029, Jiangsu Province, China

Full list of author information is available at the end of the article
}

overproliferated fibrous tissue and immature trabecular bone (Boyce and Collins 2020). FD progresses slowly, and FD in the craniomaxillofacial region can result in several clinical features, such as local bone swelling, deformity, and dental occlusion dysfunction (Akintoye et al. 2013). Because of the unclear pathogenesis of FD, osteoclastic suppressor-related drugs and surgical intervention are still the main forms of treatment (Feller et al. 2009). original author(s) and the source, provide a link to the Creative Commons licence, and indicate if changes were made. The images or other third party material in this article are included in the article's Creative Commons licence, unless indicated otherwise in a credit line to the material. If material is not included in the article's Creative Commons licence and your intended use is not permitted by statutory regulation or exceeds the permitted use, you will need to obtain permission directly from the copyright holder. To view a copy of this licence, visit http://creativecommons.org/licenses/by/4.0/. 
Overaccumulation of cyclic adenosine monophosphate (cAMP) in FD due to guanine nucleotide-binding protein alpha-stimulating activity polypeptide (GNAS) mutation is the key cause of FD pathogenesis (Zhang et al. 2012a; Riminucci et al. 2010). Excessive cAMP resulting from Gas signaling activates cAMP-response elementbinding protein (CREB) via cAMP-dependent protein kinase (PKA) (Mayr and Montminy 2001; Sands and Palmer 2008). Some studies have shown that aberrant gene expression is involved in the abnormal phenotype of FD BMSCs through binding of cAMP with the cAMPresponse element (CRE) in the promoter region of these genes and subsequent activation or inhibition (Boyce and Collins 2020; Fan et al. 2012; Khan et al. 2018).

MicroRNAs (miRNAs) are a class of small noncoding RNAs derived from precursors with a hairpin-like structure, and significant insight has been gained regarding their potential importance in numerous biological and pathological processes, such as cell proliferation, apoptosis and differentiation (Huang et al. 2011; Miska 2005; Frohlich 2019; Long et al. 2020). MicroRNAs are encoded in the genome and transcribed by RNA polymerase II (Pol II) (Ha and Kim 2014). Additionally, transcription factors could also bind to miRNA promoter regions to either activate or repress their transcription (Zhang et al. 2015). Most miRNAs regulate target gene expression by binding its $3^{\prime}$-untranslated region $\left(3^{\prime}\right.$ UTR) in a sequencecomplimentary manner to repress messenger RNA (mRNA) translation or facilitate mRNA degradation (Waki et al. 2016; Luan et al. 2017). Increasing evidence indicates that miRNAs participate in multiple biological processes in many diseases, and modulation of miRNAs has been used for the therapy of different disorders.

The miR-181 family, comprising miR-181a, miR-181b, miR-181c, and miR-181d, which are highly conserved sequences (Braicu et al. 2019). A growing number of studies show that miR-181a is involved in cell differentiation (Pop-Bica et al. 2018; Bhushan et al. 2013), autophagy (Rippo et al. 2014), apoptosis (Ouyang et al. 2012) and proliferation (Braicu et al. 2019). Moreover, miR-181a is reported to regulate the production of intracellular cAMP by decreasing AC9 expression (Zhuang et al. 2014) and to downregulate CREB1 expression by targeting its mRNA 3' UTR in neurons (Liu et al. 2013).

In the present study, we aimed to explore the miR181a-5p expression level in FD BMSCs and reveal that low expression of miR-181a-5p leads to increased proliferative ability, decreased apoptotic capacity, and impaired osteogenesis of FD BMSCs and increases the osteoclast differentiation potential. Furthermore, our study demonstrates that the CREB1-miR-181a-5p loop is critically involved in the pathologic mechanism underlying craniofacial FD. These results suggest that therapeutic intervention targeting the CREB1-miR-181a-5p loop might be beneficial for offering novel insight into the clinical treatment of FD.

\section{Methods \\ Primary cell culture}

All experiments were performed under a protocol approved by the Ethics and Research Committee of Nanjing Medical University; fresh FD tissues were immediately obtained from bone lesions after surgical removal. As a control, normal jaws were harvested from the maxilla alveoli as previously described (Xiao et al. 2019). Informed consent was obtained before volunteers were enrolled in this study. Primary BMSCs were cultured in $25 \mathrm{~cm}^{2}$ flasks with standard medium consisting of Dulbecco's modified Eagle's medium (DMEM) (Gibco, Grand Island, NY, USA), $100 \mathrm{U} / \mathrm{ml}$ penicillin and $100 \mu \mathrm{g} / \mathrm{ml}$ streptomycin, and $10 \%$ fetal bovine serum (FBS) (ScienCell, Carlsbad, CA, USA) at $37{ }^{\circ} \mathrm{C}$ maintained in $5 \% \mathrm{CO}_{2}$. The medium was changed every 3 days until $80-90 \%$ confluence was achieved. BMSCs from FD lesions and normal jaws were used at passages 3-5 throughout the experiments.

\section{miRNA sequencing analysis}

Total RNA from BMSCs and FD BMSCs was extracted using TRIzol reagent (Takara, Dalian, China) and used for miRNA sequencing analysis (Aksomics, China).

\section{KEGG analysis of target genes}

Potential target genes of miR-181a-5p were predicted using the TargetScan, miRWalk, miRPathDB and MiRDB databases, with the Micro- $\mathrm{T}$ threshold set at 0.8 and the $\mathrm{P}$-value threshold set at 0.05 . The common target genes subsequently underwent Kyoto Encyclopedia of Genes and Genomes (KEGG) database analysis to identify the enriched pathways that might be involved.

miRNA mimics/inhibitor transfection and RNA interference Cells were cultured in 12-well plates for transfection at a concentration of $8 \times 10^{4}$ cells per well. MiR-181a-5p mimics, inhibitor and control (miR NC) were purchased from GenePharma (Shanghai, China) and transfected into cells using Lipofectamine 2000 (Invitrogen, Carlsbad, CA, USA). A final concentration of $20 \mathrm{nmol} / \mathrm{l}$ miRNA was used for miRNA transfection. Small interfering RNA (siRNA) targeting CREB1 and scramble siRNA were purchased from GenePharma (Shanghai, China). The CREB1 siRNA sequence, 5'-GCCACAGAUUGCCACAUUATT3', 5'-UAAUGUGGCA AUCUGUGGCTT-3', followed our previous study (Xiao et al. 2019), and the scramble siRNA sequence was 5'-UUCUCCGAACGUGUCACG UTT-3', 5' -ACGUGACACGUUCGGAGAATT-3'. 


\section{cAMP extraction and measurement}

For measurement of intracellular levels of cAMP, the cells were incubated for $1 \mathrm{~h}$ in serum-free medium containing $1 \mathrm{mM}$ 3-isobutyl-1-methylxanthine (Sigma, St. Louis, MO, USA). cAMP was extracted by $0.1 \mathrm{M} \mathrm{HCl}$ for $20 \mathrm{~min}$, and the lysate was centrifuged at $1000 \times g$ for $10 \mathrm{~min}$ at $4{ }^{\circ} \mathrm{C}$. The supernatant was collected for measurement of cAMP levels using a Cyclic AMP ELISA Kit (Cayman, Ann Arbor, MI, USA) according to the manufacturer's instructions. The results were expressed as picomoles/milligram protein ( $\mathrm{pmol} / \mathrm{mg}$ protein).

\section{Osteoblast differentiation and alizarin red staining}

Cells were cultured in complete medium supplemented with $50 \mu \mathrm{M}$ ascorbic acid (Sigma, St. Louis, MO, USA), $10 \mathrm{mM} \beta$-glycerophosphate (Sigma), and $10^{-7} \mathrm{M}$ dexamethasone (Sigma). The medium was changed every 3 days. The mineralization potential was assessed via alizarin red staining when cells were cultured in osteogenic medium for 14 days. For alizarin red staining, the cells were fixed in anhydrous alcohol for $30 \mathrm{~min}$ and washed with double-distilled $\mathrm{H}_{2} \mathrm{O}$. Subsequently, the cells were stained with $2 \%$ Alizarin Red S ( $\mathrm{pH}$ 4.2) (Sigma) for $10 \mathrm{~min}$. To quantify nodule mineralization, calcified nodules were eluted with $10 \%$ cetylpyridinium chloride (CPC) (Sigma), and the absorbance at $562 \mathrm{~nm}$ was compared to calcium standards.

\section{Osteoclast differentiation}

Conditioned medium was collected from cell cultures. The medium was collected after the cells reached confluence for $24 \mathrm{~h}$, briefly centrifuged and then stored at $-80{ }^{\circ} \mathrm{C}$. Bone marrow mononuclear cells (BMMCs) were obtained as described previously (Zhu et al. 2019; Wijekoon et al. 2018). BMMCs were seeded in 48-well plates (Corning, New York, USA) at a density of $10^{5}$ cells/ well and cultured in $\alpha$-MEM (Gibco) containing $20 \mathrm{ng} /$ $\mathrm{ml}$ recombinant human $\mathrm{M}-\mathrm{CSF}$ (R\&D Systems, USA) and 10\% FBS for 3 days. After 3 days, nonadherent cells were removed by washing, and adherent cells were further cultured in $80 \% \alpha$-MEM supplemented with $20 \mathrm{ng} /$ $\mathrm{ml}$ M-CSF, $50 \mathrm{ng} / \mathrm{ml}$ recombinant human RANKL (R\&D Systems), 10\% FBS and 20\% conditioned medium obtained from cell culture supernatants for 10 days. The culture media were changed every three days (Hong et al. 2014).

\section{Western blot}

Western blot was performed as previously described (Xu et al. 2016). Briefly, cells were lysed, and the lysate was separated on 10\% SDS-PAGE gels and subsequently transferred to PVDF membranes (Millipore, Billerica,
MA, USA). The membranes were blocked in 5\% fat-free milk for $2 \mathrm{~h}$ and subsequently incubated with different primary antibodies (1:1000 dilution) at $4{ }^{\circ} \mathrm{C}$ overnight. Detailed information regarding the primary antibodies is listed in Additional file 1: Table S1. After washing, the membranes were incubated with secondary antibodies (1:5000 dilution). The proteins were detected by ImageQuant LAS 4000 (GE, USA). The expression levels were normalized to those of GAPDH. Quantitative analysis of the western blot was carried out using ImageJ software.

\section{RNA extraction and quantitative real-time PCR}

Total RNA from cells was extracted using TRIzol reagent (Takara) according to the manufacturer's instructions. Quantitative real-time PCR analyses were performed in triplicate using SYBR Green PCR Master Mix (Vazyme, Nanjing, Jiangsu, China), and reactions were detected using an Applied Biosystems 7900HT Fast Real-time PCR system (Applied Biosystems, Gaithersburg, CA, USA). The primer sequences used for quantitative realtime PCR are listed in Additional file 2: Table S2. The expression levels of mRNA were normalized to GAPDH. The primer of miR-181a-5p, miR-145-3p, miR-98-3p, miR-92b-5p and U6 were purchase from GeneCopoeia (Guangzhou, China) and All-in-One ${ }^{\mathrm{TM}}$ miRNA RT-qPCR Detection kit (GeneCopoeia) was used to analyze miRNAs expression, with U6 small nucleolar RNA as an internal control.

\section{Tartrate-resistant acid phosphate (TRAP) staining}

Cells were subjected to TRAP staining using a kit (Sigma) following the manufacturer's instructions. Cells containing more than 3 TRAP-positive nuclei were considered multinuclear osteoclasts and were counted by three independent assessors.

\section{F-actin ring formation assay}

Multinuclear osteoclasts were fixed with $4 \%$ formaldehyde for $30 \mathrm{~min}$ and permeabilized with $0.2 \%$ Triton X-100 for $5 \mathrm{~min}$. The cells were then blocked with $1 \%$ goat serum and 3\% BSA and incubated with $2 \mathrm{U} / \mathrm{ml}$ rhodamine phalloidin (Beyotime, Shanghai, China) (1:1000 dilution) at room temperature for $30 \mathrm{~min}$. Cell nuclei were stained with $1 \mu \mathrm{g} / \mathrm{ml}$ DAPI (Beyotime) for $1 \mathrm{~min}$. Cells were visualized using a fluorescence microscope.

\section{Bromodeoxyuridine assay and immunofluorescence}

Cells were seeded in 24-well at a density of $10^{4}$ cells per well and cultured until density reached $50 \%$. Then cells were incubated for $24 \mathrm{~h}$ with $0.03 \mathrm{mg} / \mathrm{ml}$ bromodeoxyuridine (BrdU) (Sigma) and fixed with $4 \%$ paraformaldehyde for $30 \mathrm{~min}$. Fixed cells were incubated for $30 \mathrm{~min}$ in $2 \mathrm{M} \mathrm{HCl}, 15 \mathrm{~min}$ in $0.1 \mathrm{M}$ boric acid, $15 \mathrm{~min}$ in $0.5 \%$ 
Triton X-100, blocked for $1 \mathrm{~h}$ with normal goat serum at $37{ }^{\circ} \mathrm{C}$, and incubated with an anti-BrdU antibody (1:300 dilution, Proteintech, USA) at $4{ }^{\circ} \mathrm{C}$ overnight according to the manufacturer's instructions. The cells were then incubated for $40 \mathrm{~min}$ with a secondary antibody labeled with Cy3 (1:50 dilution, ABclonal, China) and stained with DAPI. The percentage of BrdU-positive cells in six randomly selected fields was determined using a microscope. The immunofluorescence staining assay was performed as we described previously (Liu et al. 2019).

\section{Chromatin immunoprecipitation (ChIP) analysis}

ChIP analysis was carried out using EZ-ChIP (Millipore) according to the manufacturer's protocol. Briefly, FD BMSCs were cross-linked with fresh formaldehyde at a final concentration of $1 \%$ at room temperature. Glycine was used to terminate the process. Then, the cells were lysed in SDS buffer and sonicated to shear the DNA at $4{ }^{\circ} \mathrm{C}$. Lysates diluted with ChIP dilution buffer were immunoprecipitated with an anti-CREB1 antibody, and a negative rabbit IgG antibody (Proteintech) was used as an internal control. Reverse-crosslinked DNA was transferred and purified and quantified by quantitative real-time PCR analysis. The primer sequences used for quantitative real-time PCR are listed in Additional file 3: Table S3.

\section{Dual luciferase reporter assay}

The putative binding region of miR-181a-5p in the CREB1 3'UTR was amplified by PCR from genomic DNA and cloned downstream of the firefly luciferase gene (FL) in the $p G L 3$-basic luciferase reporter vector (Genecopoeia, Guangzhou, Guangdong, China). For the luciferase reporter assay, 293T cells were cotransfected with individual $p G L 3-m i R$ reporter vectors (wild type or site mutated plasmid) and miR-181a-5p mimics or scramble control (miR NC) using Lipofectamine 2000 for 48 h. Cell lysates were collected and assayed with a Dual Luciferase Assay kit (Promega, Madison, WI, USA) following the manufacturer's instructions. The pRL Renilla luciferase (RL) reporter was used as an internal control. The results are displayed as the ratio of FL/RL activity.

\section{Statistical analysis}

All data examined are presented as the mean \pm S.E.M. values. All experiments were repeated independently at least three times. The statistical significance of differences between groups was calculated using Student's t-test. $P<0.05$ was considered significant.

\section{Results}

Identification of downregulated miR-181 a-5p in FD BMSCs Aiming to screen the possible miRNAs implicated in FD, we measured the expression of miRNAs by miRNA sequencing and found that miR-181a-5p was expressed at significantly lower levels in FD BMSCs than in normal BMSCs (fold $>10, P<0.05$ ) (Fig. 1a), whereas miR-145-3p and miR-98-3p were downregulated 9.1 folds and 9 folds in FD BMSCs, respectively. Quantitative real-time PCR further verified this result (Fig. 1b). Based on our previous study, we treated normal BMSCs with $2 \mathrm{mM}$ dibutyryl cAMP (cAMP) (Selleck, Shanghai, China) or $1 \mathrm{mM}$ 3-isobutyl-1-methylxanthine (IBMX) (Sigma), a broadspectrum phosphodiesterase inhibitor, to increase intracellular cAMP expression to imitate the pathological process of FD BMSCs, and downregulated expression of miR-181a-5p was detected (Fig. 1c-e). Moreover, miR145a-3p expression was also downregulated with cAMP or IBMX treatment, while miR-98-3p and miR-92b-5p were increased (Fig. 1d, e). To study the effect of miR181a-5p on miR-145-3p, miR-98-3p and miR-92b-5p, FD BMSCs and BMSCs were transfected with miR-181a-5p mimics and inhibitor, respectively. Quantitative real-time PCR results showed that miR-145-3p had no change with miR-181a-5p mimics or inhibitor treatment, whereas miR-98-3p expression showed a positive correlation with miR-181a-5p abundance, and miR-92b-5p indicated an opposite result (Fig. 1f, g). To explore the possible function of miR-181a-5p in FD, we predicted potential target genes of miR-181a-5p by TargetScan, miRWalk, miRPathDB and MiRDB and found that 459 genes were shared in the above four databases (Fig. 1h). Furthermore, we performed KEGG analysis of the 459 target genes, and the results showed that miR-181a-5p might be involved in several pathways, including the cAMP signaling pathway, apoptosis and osteoclast differentiation

\footnotetext{
(See figure on next page.)

Fig. 1 MiR-181a-5p is expressed at low levels in FD BMSCs. a MicroRNA sequencing analysis showed the differentially expressed miRNAs between FD BMSCs and BMSCs. b Quantitative real-time PCR analysis was used to detect miR-181a-5p, miR-145-3p, miR-98-3p and miR-92b-5p expression between FD BMSCs and BMSCs. c CAMP levels in BMSCs treated with CAMP or IBMX were analyzed by ELISA. MiR-181a-5p, miR-145-3p, miR-98-3p and miR-92b-5p expression in BMSCs treated with CAMP (d) or IBMX (e) was analyzed by quantitative real-time PCR. $\mathbf{f}$ Quantitative real-time PCR analysis was used to detect miR-145-3p, miR-98-3p and miR-92b-5p expression in FD BMSCs transfected with miR-181a-5p mimics. g Quantitative real-time PCR analysis was used to detect miR-145-3p, miR-98-3p and miR-92b-5p expression in BMSCs transfected with miR-181a-5p inhibitor. $\mathbf{h}$ Venn diagram showing the number of miR-181a-5p target genes predicted by performing miRDB, miRPathDB, miRWalk, and TargetScan algorithms. i KEGG analysis of the target genes of miR-181a-5p. The data are presented as the mean \pm S.E.M. values $(n \geq 3)$. ${ }^{*} P>0.05 ;{ }^{*} P<0.05 ;{ }^{* * *} P<0.001$
} 


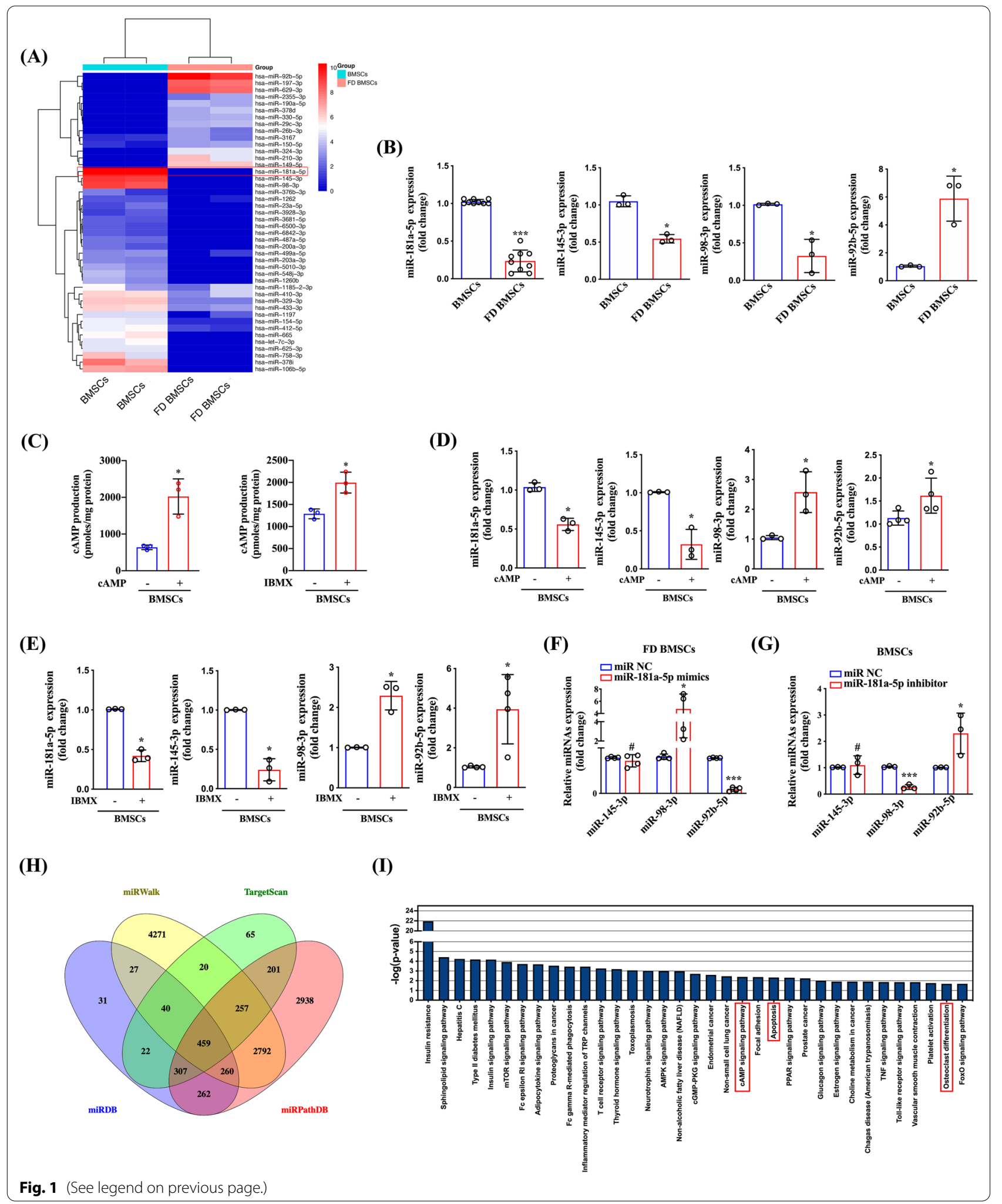




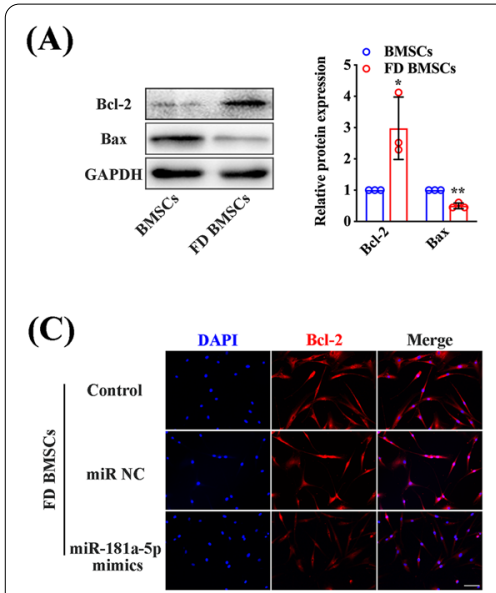

(F)

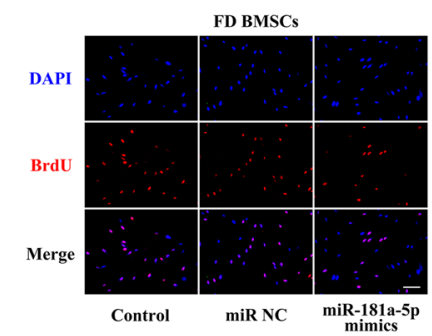

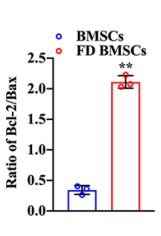

(D)
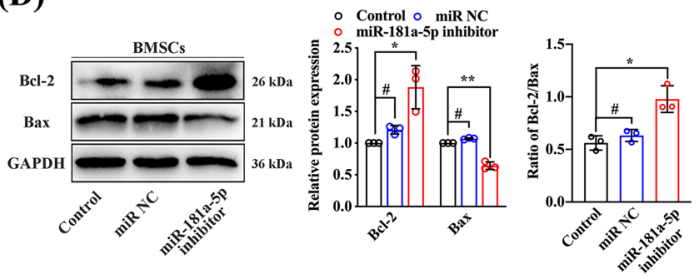

(E)

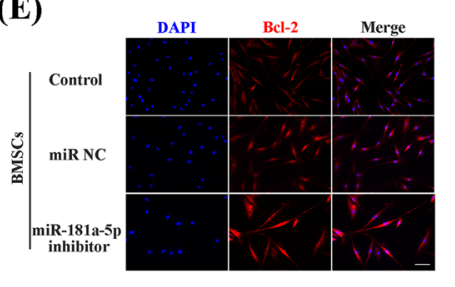

(G)

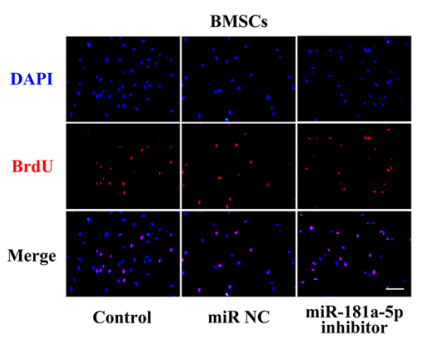

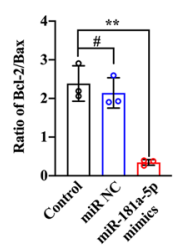

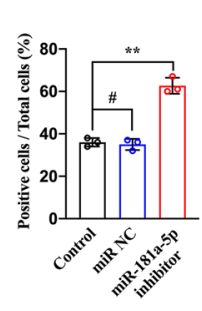

Fig. 2 Low miR-181a-5p expression suppresses the apoptosis and induces the proliferation of FD BMSCs. a Protein expression of Bcl-2 and Bax in BMSCs and FD BMSCs was detected by western blot. b Western blot analysis of BCl-2 and Bax in FD BMSCs transfected with miR-181a-5p mimics. c Immunofluorescence analysis of Bcl-2 expression in FD BMSCs transfected with miR-181a-5p mimics. $\mathbf{d}$ Western blot analysis of BCl-2 and Bax in BMSCs transfected with the miR-181a-5p inhibitor. e Immunofluorescence analysis of Bcl-2 expression in BMSCs transfected with the miR-181a-5p inhibitor. Representative BrdU incorporation in FD BMSCs transfected with miR-181a-5p mimics (f) and BMSCs transfected with the miR-181a-5p inhibitor $(\mathbf{g})$. Scale bar, $100 \mu \mathrm{m}$. The data are presented as the mean \pm S.E.M. values $(n=3) .{ }^{\#} P>0.05 ;{ }^{*} P<0.05 ;{ }^{* *} P<0.01$

(Fig. 1i). These results indicate that miR-181a-5p is expressed at low levels in FD BMSCs and may participate in the pathophysiologic process of FD.

\section{Low miR-181a-5p expression suppresses the apoptosis and promotes the proliferation of FD BMSCs}

Based on our previous study, FD BMSCs exhibited stronger proliferation ability and weaker apoptotic capacity than BMSCs (Xiao et al. 2019). We compared the expression of Bcl-2, a target gene of miR-181a, between FD BMSCs and BMSCs, and upregulated expression of $\mathrm{Bcl}-2$ and downregulated expression of Bax were detected (Fig. 2a). Moreover, the Bcl-2/Bax ratio was increased in FD BMSCs (Fig. 2a). To verify the role of miR-181a-5p in the apoptosis of FD BMSCs, cells were transfected with miR NC and miR-181a-5p mimics. Bcl-2 downregulation and Bax upregulation, with a decreased Bcl-2/Bax ratio, were observed in FD BMSCs transfected with miR-181a-5p mimics, which further confirmed apoptotic cell death (Fig. 2b). Immunofluorescence analysis also displayed the inhibited expression of Bcl-2 with miR-181a-5p overexpression (Fig. 2c). Moreover, we transfected BMSCs with a miR-181a-5p inhibitor to imitate FD BMSCs, and Bcl-2 expression and the Bcl-2/ Bax ratio were increased (Fig. 2d, e). In addition, diminished BrdU incorporation was detected in FD BMSCs transfected with miR-181a-5p mimics, and the opposite results were observed in BMSCs treated with the miR181a-5p inhibitor (Fig. 2f, g). These results suggest that downregulated miR-181a-5p suppresses apoptosis and promotes proliferation in FD BMSCs.

\section{Low expression of miR-181a-5p promotes osteogenic differentiation of BMSCs in FD}

As our previous research reported, FD BMSCs exhibit weaker osteogenic capability than normal BMSCs (Xiao et al. 2019). In this study, the same result was shown (Fig. 3a-c). To confirm whether miR-181a-5p plays a role in osteogenesis, we transfected miR-181a-5p mimics into FD BMSCs and induced osteogenesis. FD BMSCs treated with miR-181a-5p mimics showed increased expression of osteogenic markers and increased calcium deposition formation (Fig. 3d, e), while opposite trends were noted after miR-181a-5p was inhibited in normal BMSCs 


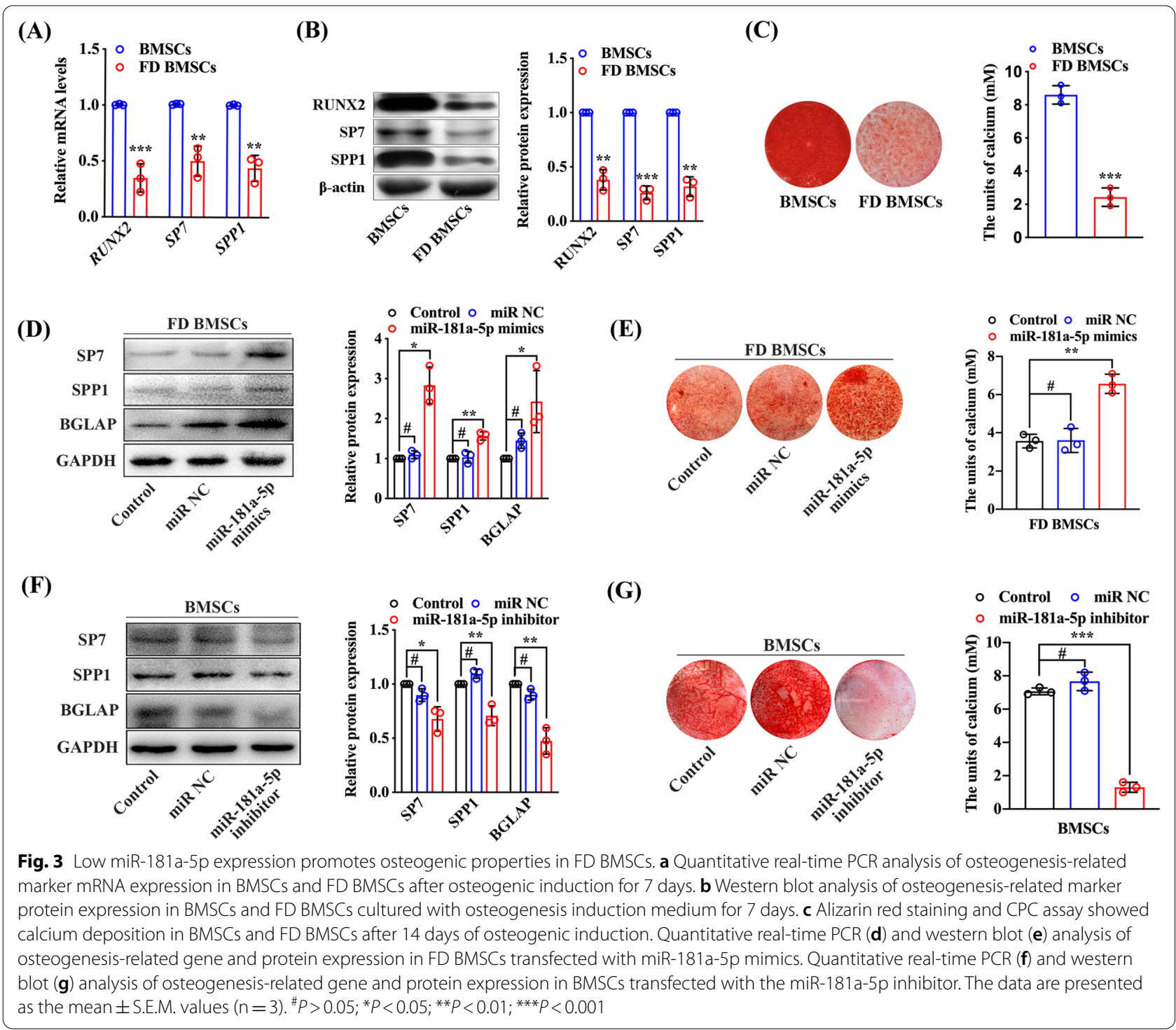

(Fig. 3f, g). These results suggest that low miR-181a-5p expression impedes ossification of BMSCs in FD.

\section{Low miR-181a-5p expression in FD BMSCs boosts osteoclast differentiation}

Fibrous actin (F-Actin) rings are the characteristic cytoskeletal structures of osteoclasts and are essential for osteoclasts involving bone resorption (Jin et al. 2019). We compared the induced osteoclast differentiation between FD BMSCs and normal BMSCs, and the results exhibited more TRAP-positive multinucleated cells and more F-Actin ring formation in the FD BMSC supernatant group (Fig. 4a, b). Moreover, increased osteoclast differentiation markers were detected in the FD BMSC supernatant group (Fig. 4c, d). To identify the role of miR-181a-5p in osteoclast differentiation and formation, the supernatant of FD BMSCs transfected with miR-181a-5p mimics was collected and used to culture osteoclasts with RANKL and M-CSF. Decreased numbers of TRAP-positive multinucleated cells and fewer F-Actin rings were detected under miR-181a-5p mimic treatment (Fig. 4e, f), and the expression of osteoclast differentiation markers was also inhibited (Fig. 4g, h). Furthermore, miR-181a-5p inhibitor-treated BMSCs led to the opposite results (Fig. 4i-l). These results indicate that downregulated miR-181a-5p in FD BMSCs promotes osteoclast differentiation and formation, in turn boosting osteoclast activity. 
(A)
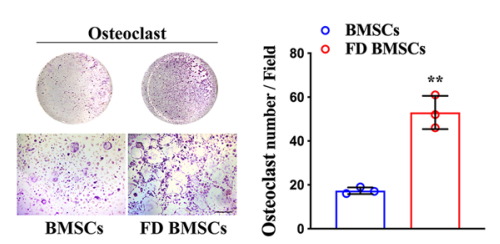

(B)

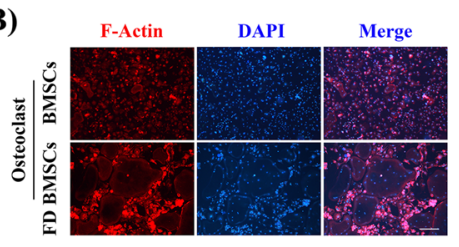

(C)

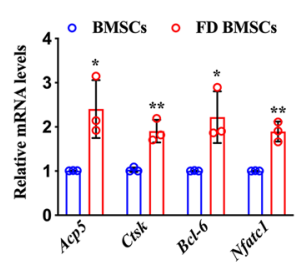

(D)

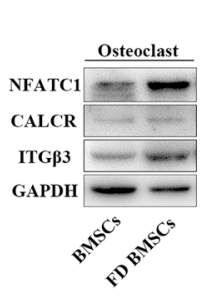

(G)

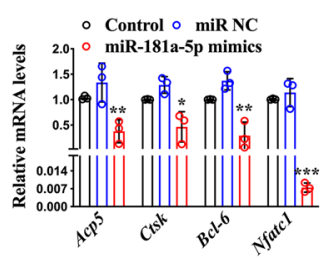

(E)

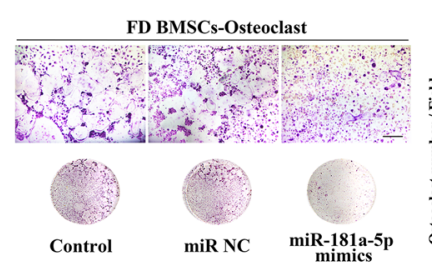

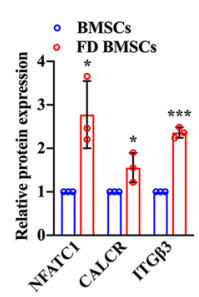

(H)

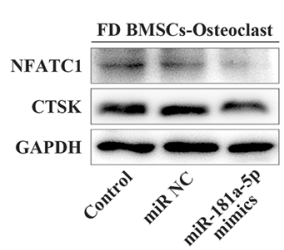

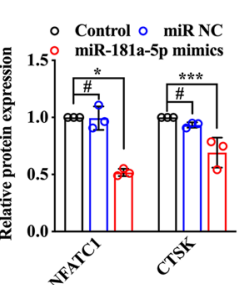

(K)

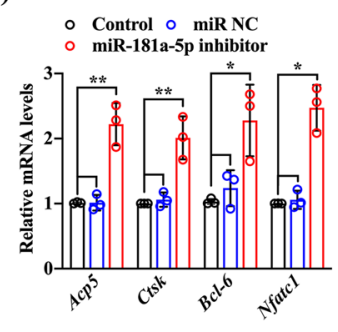

(F)

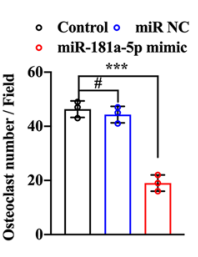

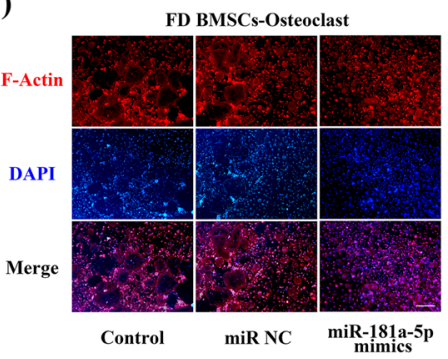

(J)

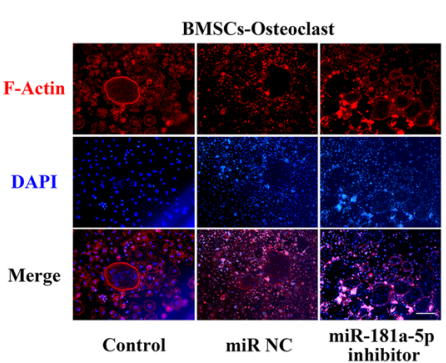

(I)
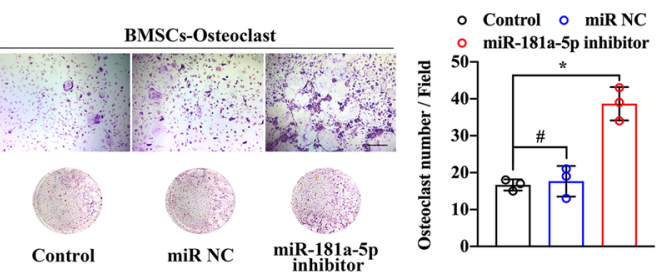

(L)
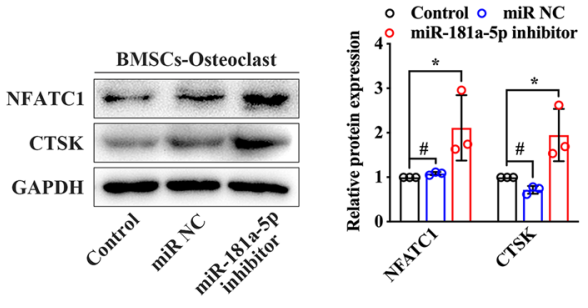

Fig. 4 Low miR-181a-5p expression promotes FD BMSC-induced osteoclast differentiation and formation. a BMSC-and FD BMSC-induced osteoclast differentiation was analyzed by TRAP staining. $\mathbf{b}$ Representative image of the F-Actin ring structure in osteoclasts. Osteoclast differentiation-related gene expression was detected by quantitative real-time PCR (c) and western blot (d). e Effect of miR-181a-5p mimics on FD BMSC-induced osteoclast differentiation was analyzed by TRAP staining. $\mathbf{f}$ Representative image of the F-Actin ring structure in osteoclasts under miR-181a-5p mimics treatment. Osteoclast differentiation-related gene expression was detected by quantitative real-time PCR (g) and western blot (h) in osteoclasts induced by FD BMSCs transfected with miR-181a-5p mimics. i Effect of the miR-181a-5p inhibitor on BMSC-induced osteoclast differentiation was analyzed by TRAP staining. $\mathbf{j}$ Representative image of the F-Actin ring structure in osteoclasts under miR-181a-5p mimics treatment. Osteoclast differentiation-related gene expression was detected by quantitative real-time PCR (k) and western blot analysis (I) in osteoclasts induced by BMSCs transfected with miR-181a-5p inhibitor. Scale bar, $100 \mu \mathrm{m}$. The data are presented as the mean \pm S.E.M. values $(\mathrm{n}=3)$. ${ }^{\#} P>0.05 ;{ }^{*} P<0.05 ;{ }^{* *} P<0.01 ;{ }^{* *} P<0.001$

\section{CREB1 interacts with miR-181a-5p by a feedback loop in FD BMSCs}

Based on the above observations, low expression of miR-181a-5p was detected in FD BMSCs, and exogenous cAMP suppressed miR-181a-5p expression in normal BMSCs. To confirm whether cAMP affected miR-181a-5p expression via CREB1, we used CREB1targeting siRNA to transfect FD BMSCs (Fig. 5a). After
CREB1-targeting RNAi, miR-181a-5p expression significantly increased (Fig. 5b). To further explore the regulatory roles of CREB1 on miR-181a-5p, we screened the miR-181a promoter region and found that CREB1 might bind to three putative binding sites (CREs) in the region from -1399 to $-578 \mathrm{bp}$. ChIP assay with a specific anti-CREB1 construct and three primers covering the miR-181a promoter region were performed. 
(A)

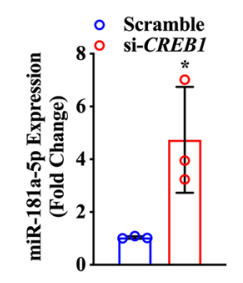

(E)

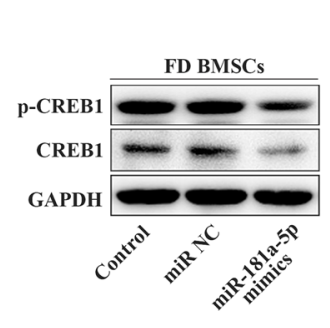

(B)

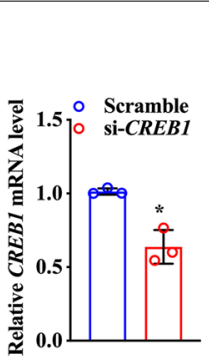

(C)

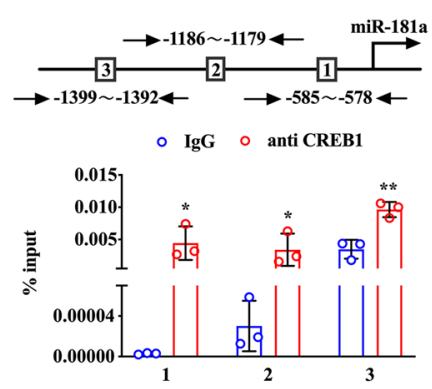

(D)

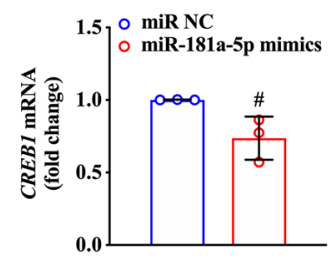

(F)

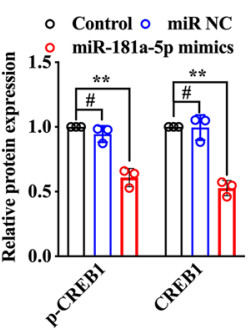

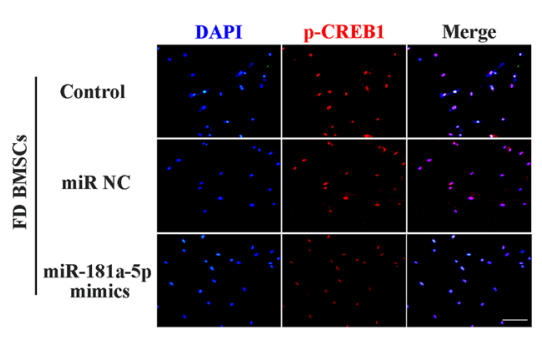

(G)

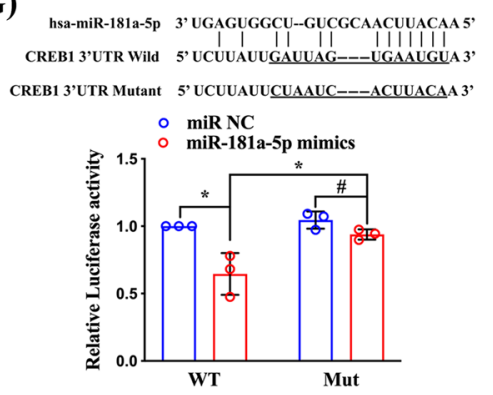

Fig. 5 CREB1 interacts with miR-181a-5p through a feedback loop in FD BMSCs. After si-CREB1 treatment for $48 \mathrm{~h}, \mathrm{CREB} 1$ (a) and miR-181a-5p (b) in FD BMSCs were detected by quantitative real-time PCR. c Three primers were designed to cover the miR-181a promoter region and were used to identify CREB1 binding sites in the ChIP assay. All three putative CREB1 binding sites in the miR-181a promoter region were identified. $\mathbf{d}$ CREB1 mRNA expression was detected by real-time PCR in FD BMSCs transfected with miR-181a-5p mimics. e Protein expression of CREB1 and $p$-CREB1 was detected by western blot. $\mathbf{f}$ Immunofluorescence analysis of $p$-CREB1 expression in FD BMSCs transfected with miR-181a-5p mimics. g Effect of miR-181a-5p on a dual-luciferase reporter plasmid bearing wild-type (WT)/mutated (Mut) CREB1 binding sites was analyzed. Cells were cotransfected with either WT-CREB1 or Mut-CREB1 and miR-181a-5p mimics or miR NC. Firefly and Renilla luciferase activities were measured in cell lysates. Scale bar, $100 \mu \mathrm{m}$. The data are presented as the mean \pm S.E.M. values $(\mathrm{n}=3) .{ }^{*} P<0.05 ;{ }^{* *} P<0.01 ;{ }^{\#} P>0.05$

Significant enrichment of CREB1 was observed at all three putative binding sites in the miR-181a promoter region (Fig. 5c). Furthermore, we transfected miR-181a-5p mimics into FD BMSCs and found that the CREB1 mRNA level had no change, whereas the p-CREB1 protein level was decreased after CREB1 reduction (Fig. 5d, e). Immunofluorescence analysis also displayed the downregulation of p-CREB1 with miR-181a-5p overexpression (Fig. 5f). To further verify the regulatory function of miR-181a-5p on CREB1, we constructed CREB1 luciferase reporter plasmids encoding the predicted 3'UTR of CREB1 mRNA in wild-type (WT) or site-mutated (Mut) forms. The reporter was cotransfected with miR NC or miR-181a-5p mimics into $293 \mathrm{~T}$ cells. The results from the luciferase reporter assay indicated that the luciferase activity significantly decreased in cells with the reporter comparable to that of miR NC (Fig. 5g). Collectively, these findings indicate that CREB1 inhibits miR-181a transcription by directly binding to its promoter region, while miR181a-5p binds to CREB1 through a posttranscriptional repression mechanism and further verify that CREB1 interacts with miR-181a-5p by a feedback loop in FD BMSCs.

\section{Discussion}

Fibrous dysplasia (FD) is a slow-progressing BMSCrelated disease with the main pathological feature being the replacement of normal bone tissue by immature fibrous trabeculae (Michienzi et al. 2007; Robey et al. 2007). FD in cranial maxillofacial bones usually has no obvious symptoms in the incipient stage. With progression of the disease, local swelling deformity, occlusal disorder, pathological fracture, local pain and other symptoms often present clinically. Mutations in GNAS are the critical cause of FD. We recently reported that compared to normal BMSCs, FD BMSCs demonstrate weaker apoptotic and osteogenic differentiation abilities and a stronger proliferation ability (Florenzano et al. 2019). In addition, FD BMSCs exhibit high osteoclastic activity in mouse models (Zhao et al. 2018; Castro et al. 2019). However, the detailed mechanism underlying these clinical features remains unclear.

miRNAs are endogenous noncoding small RNA molecules that are cut from miRNA precursors (pre-miRNAs). Mature miRNAs can form complexes with RISC and then bind to target mRNA sites through base pairing to regulate target gene expression (Treiber et al. 2019). In mammals, miRNAs are not completely complementary 
to the mRNA 3'UTR of target genes to inhibit their protein expression at the translation level (Gebert and MacRae 2019). Existing studies have shown that miRNAs are involved in a series of biological processes, such as cell proliferation and differentiation. A recent report identified a number of circulating miRNAs that are potential negative regulators of gene expression in bone cell progenitors and were associated with FD (Legrand et al. 2020). In this study, we performed miRNA sequencing analysis of normal BMSCs and FD BMSCs, and the results showed that miR-181a-5p, miR-145-3p, miR98-3p and miR-92b-5p were significantly differentially expressed miRNAs. Down-regulated expression of miR181a-5p and miR-145-3p and up-regulated expression of miR-92b-5p with cAMP or IBMX treatment suggested the possible relationship between either miR-181a-5p, miR-145-3p or miR-92b-5p and FD, while up-regulated of miR-98-3p might cause by other regulatory mechanism. Since miR-181a-5p was found that associating with apoptosis, osteogenic differentiation and osteoclast differentiation, we selected miR-181a-5p, which was the most significant downregulated miRNA in FD BMSCs (fold $>10, P<0.05$ ), and further explored its function in FD (Bhushan et al. 2013; Ouyang et al. 2012; Wang et al. 2014).

miR-181a belongs to a very conserved miRNA family. Previous studies have shown that miR-181 family members can complement multiple target miRNAs and participate in the regulation of pathophysiological processes of various diseases (Braicu et al. 2019; Seoudi et al. 2012) (See Footnote 1). Recent studies have found that miR181a can induce apoptosis in senescent cells by inhibiting the protein expression of the target gene B-cell leukemia 2 (Bcl-2) (Rippo et al. 2014). In addition to this activity in senescent cells, miR-181a also promotes apoptosis in other cells by regulating Bcl-2 (Zhu et al. 2012; Chen et al. 2010). A large number of studies have shown that mitochondrial pathways play a crucial role in apoptosis, among which Bcl-2 and BCL2-associated X (Bax), a member of the Bcl-2 family, are important regulatory genes that play an opposite role in the process of apoptosis (Adams and Cory 2007; Garner et al. 2019; Cheng et al. 1997). A decrease in the Bcl-2/Bax ratio was associated with apoptotic cell death with activation of caspase- 3 and cleavage of PARP (Karna et al. 2009). An increased $\mathrm{Bcl} 2 / \mathrm{Bax}$ ratio contributes to enhanced survival and proliferation (Zhang et al. 2012b). In the current study, Bcl-2 overexpression was detected in FD BMSCs, while Bax was downregulated. Studies have confirmed that the formation of the $\mathrm{Bcl}-2 / \mathrm{Bax}$ heterodimer can inhibit the occurrence of apoptosis by inhibiting the activation of downstream caspase-3 (Cheng et al. 1997). Considering the lower expression levels of activated caspase-3 and PARP proteins in FD BMSCs reported in our previous study (Xiao et al. 2019), we infer that high expression of $\mathrm{Bcl}-2$ and a high $\mathrm{Bcl}-2 / \mathrm{Bax}$ ratio may be the key reason for the inhibition of FD BMSC apoptosis and further indicate that downregulated miR-181a-5p contributes to Bcl-2 upregulation in FD BMSCs.

The occurrence of FD is related to the broken balance of osteogenic and osteoclastic activities. Our previous results showed that the osteogenic differentiation and mineralization capacity of FD BMSCs were weaker than those of normal BMSCs (Xiao et al. 2019). Furthermore, we found that the number of osteoclasts induced by the FD BMSC supernatant increased and that the expression of osteoclast-related genes was enhanced, suggesting that pathological fracture in FD patients might be related to enhanced activity of osteoclasts. In terms of bone metabolism, miR-181a can regulate BMP-induced osteogenic differentiation of MC3T3 cells by inhibiting the TGFbeta pathway (Bhushan et al. 2013), and miR-181a has an inhibitory effect on osteoclast survival (Wang et al. 2014). Interestingly, miR-181a promoted osteoclast apoptosis by regulating FasL protein expression (Shao et al. 2015). Our study showed that FD BMSCs treated with exogenous miR-181a-5p exhibited enhanced osteogenic differentiation and mineralization abilities and weaker osteoclast activity. These data imply that a sufficient miR-181a level is an important factor in maintaining bone homeostasis.

The regulatory effect of cAMP on cells is realized by activating the downstream PKA-CREB pathway. Phosphorylated CREB forms a homodimer that binds to a class of cAMP response element (CRE) in the gene transcriptional regulatory region to regulate gene transcription. Some miRNAs may have independent transcriptional regulatory units and in the same cluster are generally co-transcribed (Ha and Kim 2014; Stavast and Erkeland 2019), and transcription factors could positively or negatively regulate miRNA expression ( $\mathrm{Ha}$ and Kim 2014). Recent studies have demonstrated that CREB1 can act with miRNAs in regulatory networks by feedback loops (Wang et al. 2016). In this study, we uncovered that miR-181a-5p was regulated by CREB1. To gain further insight into the mechanism, we analyzed the promoter region of miR-181a and identified three putative binding sites of CREB1. Our data first verified that miR181a-5p is the downstream effector of CREB1. However, the detailed mechanism remains largely unexplored and needs to be further investigated.

The regulation of target genes by miRNAs mainly occurs at the posttranscriptional level. In animals, most miRNAs regulate the expression of target genes mainly by complementing the target gene mRNA 3'UTR to suppress gene translation (Gebert and MacRae 2019). MiR181a regulates CREB1 expression by targeting its mRNA 


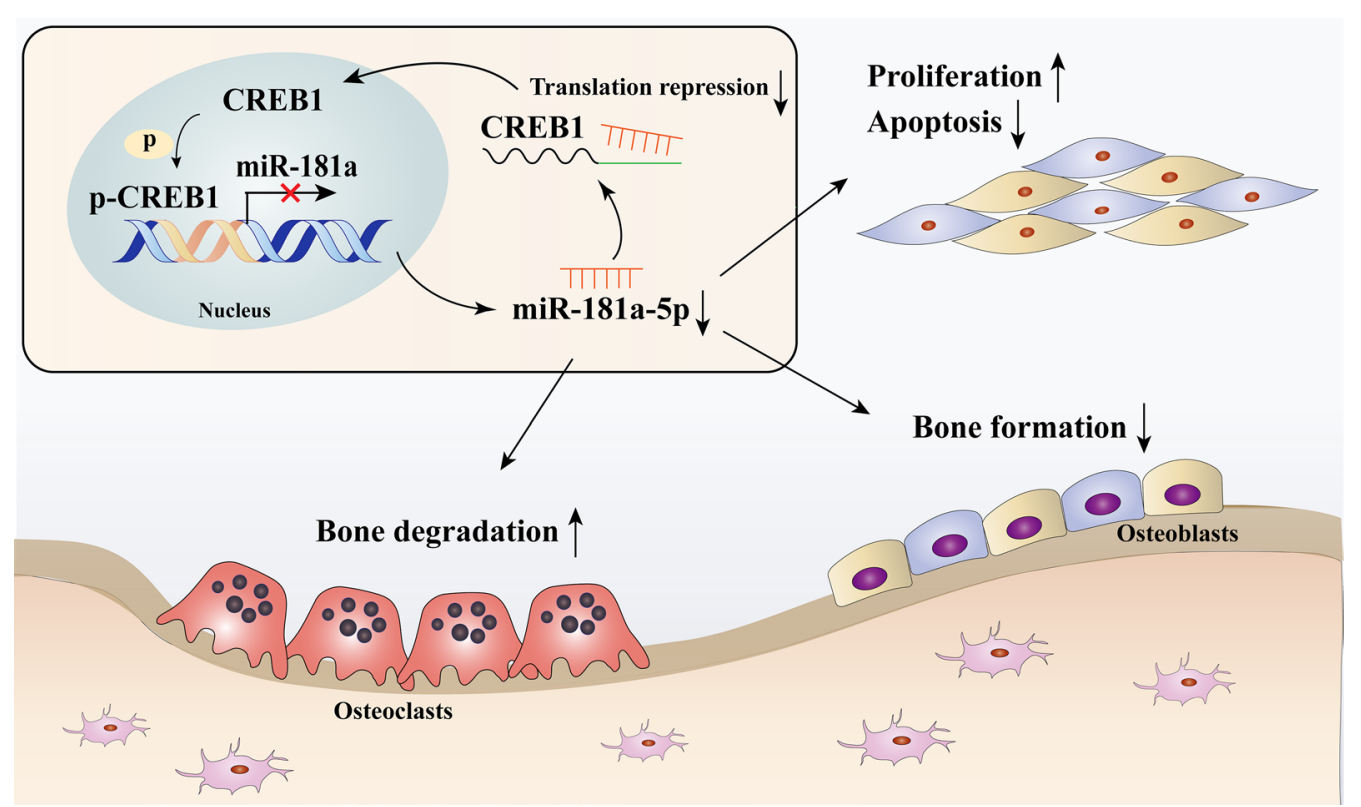

Fig. 6 The CREB1-miR-181a-5p loop in BMSCs from FD lesions. Overproduction of CAMP promotes CREB1 phosphorylation, and p-CREB1 binds to the CRE in the miR-181a promoter to suppress its transcription. Downregulation of miR-181a-5p leads to enhanced proliferation ability, decreased apoptotic capacity, and impaired osteogenic differentiation of FD BMSCs and increases the osteoclast differentiation potential, resulting in clinicopathological features of FD. Moreover, downregulation of miR-181a-5p promotes CREB1 expression by decreasing the inhibition of its translation and further exacerbates pathological manifestations of FD

3'UTR in neurons (Liu et al. 2013). In our study, we found that miR-181a-5p inhibited the protein but not the mRNA expression of CREB1 in FD BMSCs and further ruled out that miR-181a-5p regulated CREB1 expression by binding to its 3'UTR. These results suggest that miR-181a-5p has a negative regulatory effect on CREB1 expression by translation suppression. The presence of the CREB1-miR-181a-5p loop, in which CREB1 interacts with miR-181a-5p by negative feedback regulation, is critically involved in the abnormal properties of FD BMSCs.

In summary, our studies, taken together with these results, demonstrate for the first time that the CREB1miR-181a-5p loop is associated with the disordered properties of FD BMSCs (Fig. 6). Furthermore, intervention with miR-181a-5p reversed the FD phenotype to some extent. Importantly, this study provides useful insights into the molecular pathogenesis of FD and the evaluation of potential therapeutic strategies for FD in vitro.

\section{Conclusions}

The current study demonstrates that the CREB1-miR181a-5p loop participates in the pathological phenotype of FD BMSCs. In this context, our results suggest that targeted modulation of miR-181a-5p in FD BMSCs might be a potential therapeutic strategy for FD in vitro.
Continued investigation of this loop may be promising for providing a possible therapeutic strategy for FD patients.

\section{Abbreviations}

BMSCs: Bone marrow stromal cells; FD: Fibrous dysplasia; miRNA: MicroRNA; GNAS: Guanine nucleotide-binding protein alpha-stimulating activity polypeptide; CAMP: Cyclic adenosine monophosphate; CREB: CAMP-response element-binding protein; PKA: CAMP-dependent protein kinase; CRE: CAMPresponse element; mRNA: Messenger RNA; DMEM: Dulbecco's Modified Eagle Medium; FBS: Fetal bovine serum; KEGG: Kyoto Encyclopedia of Genes and Genomes; CPC: Cetylpyridinium chloride; BMMCs: Bone marrow mononuclear cells; BrdU: Bromodeoxyuridine; IBMX: 3-Isobutyl-1-methylxanthine; TRAP: Tartrate-resistant acid phosphate; ChIP: Chromatin immunoprecipitation.

\section{Supplementary Information}

The online version contains supplementary material available at https://doi. org/10.1186/s10020-021-00341-z.

Additional file 1. Table S1. The primary antibodies used in western blot. Additional file 2. Table $\mathbf{S 2}$. Sequence information on specific primers used in this study.

Additional file 3. Table S3. Sequence of ChIP primers.

\section{Authors' contributions}

YF: Conception and design, performed research, conducted data analysis and interpretation, conducted manuscript writing; ZX: Performed and revised manuscript; TX: Performed research, conducted data analysis and interpretation; ZL: Conducted data analysis and interpretation; HX: Collected and assembled data; XS: Collected and assembled data; LL: Performed research; LX: 
Collected and assembled data; $\mathrm{HJ}$ : Performed study conception and design, provided study material or patients. All authors gave final approval and agree to be accountable for all aspects of the work.

\section{Funding}

This work was supported by National Natural Science Foundation of China Grant (81500874), the Natural Science Foundation of the Jiangsu Higher Education Institutions of China (Grant No. 19KJA360003), and the Priority Academic Program for the Development of Jiangsu Higher Education Institutions (PAPD, 2018-87).

\section{Availability of data and materials}

The datasets used and/or analyzed during the current study are available from the corresponding author on reasonable request.

\section{Declarations}

\section{Ethics approval and consent to participate}

All experiments were performed under a protocol approved by the Ethics and Research Committee of Nanjing Medical University.

\section{Competing interest}

The authors declare no potential conflicts of interest with respect to the authorship and/or publication of this article.

\section{Author details}

'Jiangsu Province Key Laboratory of Oral Diseases, Nanjing Medical University, No.136, Hanzhong Road, Nanjing 210029, Jiangsu Province, China. ${ }^{2}$ Department of Oral and Maxillofacial Surgery, Affiliated Hospital of Stomatology, Nanjing Medical University, Nanjing 210029, China. ${ }^{3}$ Jiangsu Province Engineering Research Center of Stomatological Translational Medicine, Nanjing Medical University, Nanjing 210029, China. ${ }^{4}$ Department of Oral and Maxillofacial Surgery, Nanjing Stomatological Hospital, Medical School of Nanjing University, Nanjing 210008, China.

\section{Received: 21 January 2021 Accepted: 6 July 2021}

Published online: 22 July 2021

\section{References}

Adams JM, Cory S. Bcl-2-regulated apoptosis: mechanism and therapeutic potential. Curr Opin Immunol. 2007;19(5):488-96.

Akintoye SO, Boyce AM, Collins MT. Dental perspectives in fibrous dysplasia and McCune-Albright syndrome. Oral Surg Oral Med Oral Pathol Oral Radiol. 2013;116(3):e149-155.

Bhushan R, Grunhagen J, Becker J, Robinson PN, Ott CE, Knaus P. miR-181a promotes osteoblastic differentiation through repression of TGF-beta signaling molecules. Int J Biochem Cell Biol. 2013;45(3):696-705.

Boyce AM, Collins MT. Fibrous dysplasia/McCune-Albright syndrome: a rare, mosaic disease of galphas activation. Endocr Rev. 2020;41(2):345-70.

Braicu C, Gulei D, Raduly L, Harangus A, Rusu A, Berindan-Neagoe I. Altered expression of miR-181 affects cell fate and targets drug resistance-related mechanisms. Mol Aspects Med. 2019;70:90-105.

Chen G, Zhu W, Shi D, Lv L, Zhang C, Liu P, Hu W. MicroRNA-181a sensitizes human malignant glioma U87MG cells to radiation by targeting $\mathrm{BCl}-2$. Oncol Rep. 2010;23(4):997-1003.

Cheng EH, Kirsch DG, Clem RJ, Ravi R, Kastan MB, Bedi A, Ueno K, Hardwick JM. Conversion of Bcl-2 to a Bax-like death effector by caspases. Science. 1997;278(5345):1966-8.

de Castro LF, Burke AB, Wang HD, Tsai J, Florenzano P, Pan KS, Bhattacharyya N, Boyce AM, Gafni RI, Molinolo AA, et al. Activation of RANK/RANKL/ OPG pathway is involved in the pathophysiology of fibrous dysplasia and associated with disease burden. J Bone Miner Res. 2019;34(2):290-4.

Fan QM, Yue B, Bian ZY, Xu WT, Tu B, Dai KR, Li G, Tang TT. The CREB-Smad6Runx2 axis contributes to the impaired osteogenesis potential of bone marrow stromal cells in fibrous dysplasia of bone. J Pathol. 2012;228(1):45-55.

Feller L, Wood NH, Khammissa RA, Lemmer J, Raubenheimer EJ. The nature of fibrous dysplasia. Head Face Med. 2009;5(22):1-5.
Florenzano P, Pan KS, Brown SM, Paul SM, Kushner H, Guthrie LC, de Castro LF, Collins MT, Boyce AM. Age-related changes and effects of bisphosphonates on bone turnover and disease progression in fibrous dysplasia of bone. J Bone Miner Res. 2019;34:653-60.

Frohlich LF. Micrornas at the interface between osteogenesis and angiogenesis as targets for bone regeneration. Cells. 2019;8(2):121.

Garner T, Amgalan D, Reyna D, Li S, Kitsis R, Gavathiotis E. Small-molecule allosteric inhibitors of BAX. Nat Chem Biol. 2019;15(4):322-30.

Gebert LFR, MacRae IJ. Regulation of microRNA function in animals. Nat Rev Mol Cell Biol. 2019;20(1):21-37.

Ha M, Kim VN. Regulation of microRNA biogenesis. Nat Rev Mol Cell Biol. 2014;15(8):509-24.

Hong YY, Yu FY, Qu JF, Chen F, Li TJ. Fibroblasts regulate variable aggressiveness of syndromic keratocystic and non-syndromic odontogenic tumors. J Dent Res. 2014;93(9):904-10.

Huang Y, Shen XJ, Zou Q, Wang SP, Tang SM, Zhang GZ. Biological functions of microRNAs: a review. J Physiol Biochem. 2011;67(1):129-39.

Jin H, Yao L, Chen K, Liu Y, Wang Q, Wang Z, Liu Q, Cao Z, Kenny J, Tickner $J$, et al. Evodiamine inhibits RANKL-induced osteoclastogenesis and prevents ovariectomy-induced bone loss in mice. J Cell Mol Med. 2019;23(1):522-34

Karna P, Sharp SM, Yates C, Prakash S, Aneja R. EM011 activates a survivindependent apoptotic program in human non-small cell lung cancer cells. Mol Cancer. 2009;8:93.

Khan SK, Yadav PS, Elliott G, Hu DZ, Xu R, Yang Y. Induced Gnas (R201H) expression from the endogenous Gnas locus causes fibrous dysplasia by up-regulating Wnt/beta-catenin signaling. Proc Natl Acad Sci USA 2018;115(3):E418-27

Legrand MA, Millet M, Merle B, Rousseau JC, Hemmendinger A, Gineyts E, Sornay-Rendu E, Szulc P, Borel O, Croset M, et al. A signature of circulating miRNAs associated with fibrous dysplasia of bone: the mirDys study. J Bone Miner Res. 2020;35:1881-92.

Liu Y, Zhao Z, Yang F, Gao Y, Song J, Wan Y. microRNA-181a is involved in insulin-like growth factor-1-mediated regulation of the transcription factor CREB1. J Neurochem. 2013;126(6):771-80.

Liu X, Fu Y, Huang J, Wu M, Zhang Z, Xu R, Zhang P, Zhao S, Liu L, Jiang H. ADAR1 promotes the epithelial-to-mesenchymal transition and stemlike cell phenotype of oral cancer by facilitating oncogenic microRNA maturation. J Exp Clin Cancer Res. 2019;38(1):315.

Long J, He Q, Yin Y, Lei X, Li Z, Zhu W. The effect of miRNA and autophagy on colorectal cancer. Cell Prolif. 2020;53(10):e12900.

Luan X, Zhou X, Trombetta-eSilva J, Francis M, Gaharwar AK, Atsawasuwan P, Diekwisch TGH. MicroRNAs and periodontal homeostasis. J Dent Res. 2017;96(5):491-500.

Mayr B, Montminy M. Transcriptional regulation by the phosphorylationdependent factor CREB. Nat Rev Mol Cell Biol. 2001;2(8):599-609.

Michienzi S, Cherman N, Holmbeck K, Funari A, Collins MT, Bianco P, Robey $P G$, Riminucci M. GNAS transcripts in skeletal progenitors: evidence for random asymmetric allelic expression of Gs alpha. Hum Mol Genet. 2007;16(16):1921-30.

Miska EA. How microRNAs control cell division, differentiation and death. Curr Opin Genet Dev. 2005;15(5):563-8.

Ouyang YB, Lu Y, Yue S, Giffard RG. miR-181 targets multiple BCl-2 family members and influences apoptosis and mitochondrial function in astrocytes. Mitochondrion. 2012;12(2):213-9.

Pop-Bica C, Pintea S, Cojocneanu-Petric R, Del Sal G, Piazza S, Wu Z-H, Alencar AJ, Lossos IS, Berindan-Neagoe I, Calin GA. MiR-181 family-specific behavior in different cancers: a meta-analysis view. Cancer Metastasis Rev. 2018;37(1):17-32.

Riminucci M, Robey PG, Saggio I, Bianco P. Skeletal progenitors and the GNAS gene: fibrous dysplasia of bone read through stem cells. J Mol Endocrinol. 2010;45(6):355-64.

Rippo MR, Olivieri F, Monsurro V, Prattichizzo F, Albertini MC, Procopio AD. MitomiRs in human inflamm-aging: a hypothesis involving miR-181a, miR-34a and miR-146a. Exp Gerontol. 2014;56:154-63.

Robey PG, Kuznetsov S, Riminucci M, Bianco P. The role of stem cells in fibrous dysplasia of bone and the Mccune-Albright syndrome. Pediatr Endocrinol Rev. 2007;4:386-94.

Sands WA, Palmer TM. Regulating gene transcription in response to cyclic AMP elevation. Cell Signal. 2008;20(3):460-6. 
Seoudi AM, Lashine YA, Abdelaziz Al. MicroRNA-181a - a tale of discrepancies. Expert Rev Mol Med. 2012;14:e5.

Shao B, Liao L, Yu Y, Shuai Y, Su X, Jing H, Yang D, Jin Y. Estrogen preserves Fas ligand levels by inhibiting microRNA-181a in bone marrow-derived mesenchymal stem cells to maintain bone remodeling balance. FASEB J. 2015;29(9):3935-44.

Stavast CJ, Erkeland SJ. The non-canonical aspects of MicroRNAs: many roads to gene regulation. Cells. 2019;8:11.

Treiber T, Treiber N, Meister G. Regulation of microRNA biogenesis and its crosstalk with other cellular pathways. Nat Rev Mol Cell Biol. 2019;20(1):5-20.

Waki T, Lee SY, Niikura T, Iwakura T, Dogaki Y, Okumachi E, Oe K, Kuroda R, Kurosaka M. Profiling microRNA expression during fracture healing. BMC Musculoskelet Disord. 2016;17(83):1-8.

Wang S, Tang C, Zhang Q, Chen W. Reduced miR-9 and miR-181a expression down-regulates Bim concentration and promote osteoclasts survival. Int J Clin Exp Pathol. 2014;7(5):2209-18.

Wang YW, Chen X, Ma R, Gao P. Understanding the CREB1-miRNA feedback loop in human malignancies. Tumour Biol. 2016;37(7):8487-502.

Wijekoon HMS, Bwalya EC, Fang J, Kim S, Hosoya K, Okumura M. Inhibitory effects of sodium pentosan polysulfate on formation and function of osteoclasts derived from canine bone marrow. BMC Vet Res. 2018;14(1):152.

Xiao T, Fu Y, Zhu W, Xu R, Xu L, Zhang P, Du Y, Cheng J, Jiang H. HDAC8, a potential therapeutic target, regulates proliferation and differentiation of bone marrow stromal cells in fibrous dysplasia. Stem Cells Transl Med. 2019;8(2):148-61.

Xu R, Fu Z, Liu X, Xiao T, Zhang P, Du Y, Yuan H, Cheng J, Jiang H. Transplantation of osteoporotic bone marrow stromal cells rejuvenated by the overexpression of SATB2 prevents alveolar bone loss in ovariectomized rats. Exp Gerontol. 2016;84:71-9.
Zhang S, Kaplan FS, Shore EM. Different roles of GNAS and CAMP signaling during early and late stages of osteogenic differentiation. Horm Metab Res. 2012a;44(10):724-31.

Zhang R, He Y, Zhang X, Xing B, Sheng Y, Lu H, Wei Z. Estrogen receptorregulated microRNAs contribute to the $B C L 2 / B A X$ imbalance in endometrial adenocarcinoma and precancerous lesions. Cancer Lett. 2012b;314(2):155-65

Zhang HM, Kuang S, Xiong X, Gao T, Liu C, Guo AY. Transcription factor and microRNA co-regulatory loops: important regulatory motifs in biological processes and diseases. Brief Bioinform. 2015;16(1):45-58.

Zhao X, Deng P, Iglesias-Bartolome R, Amornphimoltham P, Steffen DJ, Jin Y, Molinolo AA, de Castro LF, Ovejero D, Yuan Q, et al. Expression of an active Galphas mutant in skeletal stem cells is sufficient and necessary for fibrous dysplasia initiation and maintenance. Proc Natl Acad Sci USA. 2018;115(3):E428-37.

Zhu HY, Liu MY, Hong Q, Zhang D, Geng WJ, Xie YS, Chen XM. Role of microRNA-181a in the apoptosis of tubular epithelial cell induced by cisplatin. Chin Med J (engl). 2012;125(3):523-6.

Zhu W, Xu R, Du J, Fu Y, Li S, Zhang P, Liu L, Jiang H. Zoledronic acid promotes TLR-4-mediated M1 macrophage polarization in bisphosphonate-related osteonecrosis of the jaw. FASEB J. 2019;33(4):5208-19.

Zhuang LK, Xu GP, Pan XR, Lou YJ, Zou QP, Xia D, Yan WW, Zhang YT, Jia PM, Tong $\mathrm{JH}$. MicroRNA-181a-mediated downregulation of AC9 protein decreases intracellular CAMP level and inhibits ATRA-induced APL cell differentiation. Cell Death Dis. 2014;5:e1161.

\section{Publisher's Note}

Springer Nature remains neutral with regard to jurisdictional claims in published maps and institutional affiliations.
Ready to submit your research? Choose BMC and benefit from:

- fast, convenient online submission

- thorough peer review by experienced researchers in your field

- rapid publication on acceptance

- support for research data, including large and complex data types

- gold Open Access which fosters wider collaboration and increased citations

- maximum visibility for your research: over $100 \mathrm{M}$ website views per year

At BMC, research is always in progress.

Learn more biomedcentral.com/submissions 